\section{Randomised controlled trial}

\section{Varenicline can help smokers to stop smoking by gradual reduction}

10.1136/ebmed-2015-110199

\section{Karl Fagerstrom}

Fagerstrom Consulting, Vaxholm, Sweden

Correspondence to: $\mathrm{Dr}$ Karl Fagerstrom, Fagerstrom Consulting, Vaxholm 18531, Sweden; karl.fagerstrom@swipnet.se

Commentary on: Ebbert J0, Hughes JR, West RJ, et al. Effect of varenicline on smoking cessation through smoking reduction: a randomised clinical trial. JAMA 2015;313:687-94.

\section{Context}

Around 70\% of smokers say they want to quit smoking. However, if they are asked to set a quit date 'right now', the figure comes down to around $5-10 \%$. In practice, we need to realise that there is a range of motivation for change. Smokers can be grouped into those wanting and not wanting to quit. Among those wanting to quit, some may have tried and failed so many times that they have more or less given up trying. Among those not wanting to quit, there are those who want to manage their smoking, get it under better control and reduce it. There is also a small group that do not want any change.

It has been suggested that highly motivated smokers and those relatively early in their quitting phase are the ones likely to benefit from abrupt quitting. However, more smokers can probably be helped if smokers with very low self-confidence and those wanting to reduce are given sensible and less dramatic options. ${ }^{1}$ Interestingly, studies with nicotine replacement treatment that have recruited smokers not interested in quitting but in reducing smoking found that many smokers actually stopped smoking altogether. ${ }^{2}$ Varenicline is a smoking cessation treatment considered to be effective, and is of special interest because it reduces the reward from nicotine. ${ }^{3}$

\section{Methods}

In this randomised controlled trial, 1510 smokers from 10 countries and 61 centres, who were not wanting to quit, were randomised on varenicline or placebo. They were instructed to reduce smoking by $50 \%$ at week four and by $75 \%$ at week eight and to make an attempt to quit at 12 weeks. The participants continued taking medication up to week 24 , and thereafter they were followed up for an additional 28 weeks.

\section{Findings}

In the varenicline group, $32.1 \%$ managed to be continuously abstinent during the past 10 weeks of treatment (weeks 15-24), while 6.9\% managed to do so in the placebo group, relative risk $(R R)=4$.6. From week $21-52$, the figures were $24 \%$ and $6 \%$, respectively, $R R=4$. Serious adverse events were similarly uncommon in both treatment arms. Nausea, abnormal dreams and insomnia were significantly more common in the varenicline arm.

\section{Commentary}

These findings suggest that varenicline may be suitable not only for smokers interested in abrupt quitting but also for smokers not willing to stop abruptly. Thus, it is likely that, with this alternative, more smokers can be engaged in activities leading to complete cessation. Similar studies support the current finding, as does the observation from this study that the number of quitters increased up to 18 weeks.

A limitation of this study is that it is difficult to generalise results to normal practice since the smokers were given 18 clinic visits. It is likely that the absolute level of results will be somewhat lower in normal practice. Further research in testing effective procedures for engaging more smokers is needed. For example, it would be interesting to invite smokers (regardless of motivation to stop) and measure total quitting if randomised to: (1) the standard procedure; (2) choosing between standard procedure and reduction to quit; (3) choosing between standard procedure, reduction to quit and just managing smoking.

\section{Implication for practice}

For smokers not ready to stop abruptly, a less dramatic intervention, such as varenicline, can help to stop smoking.

Competing interests KF has consulted for Pfizer Inc.

Provenance and peer review Commissioned; internally peer reviewed.
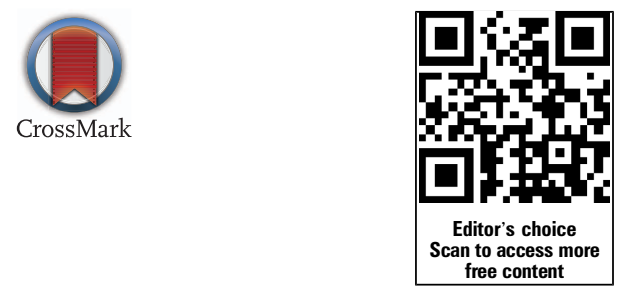

\section{References}

1. Fagerstrom K. Can reduced smoking be a way for smokers not interested in quitting to actually quit? Respiration 2005;72:216-20.

2. Lindson-Hawley N, Aveyard P, Hughes J. Reduction versus abrupt cessation in smokers who want to quit. Cochrane Database Syst Rev 2012;11:CD008033. http:// dx.doi.org/10.1002/14651858.CD008033.pub3. http://onlinelibrary.wiley.com/doi/10. 1002/14651858.CD008033.pub3/abstract

3. Cahill K, Stead LF, Lancaster T. Nicotine receptor partial agonists for smoking cessation. Cochrane Database Syst Rev 2012;4:CD006103. 